there were 32 responders and 13 non-responders. Baseline uTWEAK levels were higher in non-responder LN compared with responders LN. This was observed in both treatment either IVCY $(n=25)$ or MPS $(n=24)(175.50 \pm 36.97$ vs 57.09 $\pm 7.40 \mathrm{pg} / \mathrm{mL} ; \mathrm{p}=0.018$ or $124.90 \pm 34.53$ vs $55.69 \pm 14.22 \mathrm{pg} /$ $\mathrm{mL} ; \mathrm{p}=0.038)$. The area under the ROC curve to predict response to treatment was $0.79(95 \% \quad \mathrm{CI}=0.64-0.94)$. The cut-off level of $94.0 \mathrm{pg} / \mathrm{dL}$ predict resistant-to-treat at sensitivity and specificity of 64 and 85 percent, respectively.

Conclusions uTWEAK may be a biomarker that guide treatment of lupus nephritis patients. Targeting TWEAK protein in active lupus nephritis is an interesting choice of therapy.

\section{RAPIDLY PROGRESSIVE ILD IN IIM - THE SINGAPOREAN EXPERIENCE}

${ }^{1} \mathrm{TC}$ Tan, ${ }^{2} \mathrm{JWL}$ Tan*, ${ }^{2} \mathrm{KP}$ Leong, ${ }^{2} \mathrm{GYL}$ Chan, ${ }^{2} \mathrm{BYH}$ Thong. ${ }^{1}$ Khoo Teck Puat Hospital, General Medicine, Singapore, Singapore; ${ }^{2}$ Tan Tock Seng Hospital, Rheumatology Allergy and Immunology, Singapore, Singapore

\subsection{6/lupus-2017-000215.321}

Background and aims Interstitial lung disease (ILD) can be associated with the idiopathic inflammatory myopathies (IIM). Rapidly progressive interstitial lung disease (RP-ILD) has been recognised in Asian cohorts and has a high mortality. This study aims to describe a cohort of RP-ILD in Singapore, and identify factors associated with RP-ILD.

Methods This is a retrospective study of IIM-ILD patients in the Department of Rheumatology, Allergy and Immunology, Tan Tock Seng Hospital. Patient symptoms, clinical and serologic features, mortality were compared.

Results There were 68 IIM-ILD patients from 2003-12, 63 cases were analysed. RP-ILD was identified in 14 (22\%). The RP-ILD group was more likely to be male $(42.9 \%$ versus $24.5 \%)$, were more likely to present with fever (50\% vs $14.3 \%, \mathrm{p}=0.01)$, cough $(71.4 \%$ vs $34.7 \%, \mathrm{p}=0.03)$, and dyspnea at rest $(21.4 \%$ vs $2 \%, \mathrm{p}=0.03)$. A larger proportion of patients with RP-ILD had amyopathic dermatomyositis (ADM) (35.7\% vs $20.4 \%)$. None of the patients with RP-ILD had anti-Jo1 (0\% vs 34\%, p=0.01). All RP-ILD cases deteriorated, with $80 \%$ requiring mechanical ventilation. The mortality was $100 \%$ in the RP-ILD group (vs $16 \%$ in non RP-ILD group, $\mathrm{p}=0.00$ ). Median time from diagnosis to death was 26 days. Conclusions We identified ADM and absence of anti Jo-1 as strong associations for RP-ILD. Mortality was $100 \%$ in this RP-ILD cohort. Clinicians should have heightened awareness of this phenotype, early management at specialised respiratory care units, aggressive combination immunosuppressive therapy may be key to mortality reduction.

\section{DOWN-REGULATION OF MIR-10A INDUCES IL-8 IN HUMAN MESANGIAL CELLS STIMULATED WITH ANTI- DSDNA IGG ANTIBODIES}

\footnotetext{
${ }^{1} \mathrm{P}$ Tangtanatakul ${ }^{*},{ }^{2} \mathrm{~B}$ Thammasate, ${ }^{3} \mathrm{~A}$ Jacquet, ${ }^{2} \mathrm{R}$ Reantragoon, ${ }^{4} \mathrm{~T}$ Pisitkun, ${ }^{5} \mathrm{Y}$ Avihingsanon, ${ }^{2} \mathrm{~A}$ Leelahavanichkul, ${ }^{2} \mathrm{~N}$ Hirankarn. ${ }^{1}$ Chulalongkorn University, Microbiology, Bangkok, Thailand; '2Chulalongkorn, Microbiology, Bangkok, Thailand; ${ }^{3}$ Chulalongkorn, Research, Bangkok, Thailand; ${ }^{4}$ Chulalongkorn, Reserach, Bangkok, Thailand; ${ }^{5}$ Chulalongkorn, Medicine, Bangkok, Thailand
}

Background and aims The objective of this study is to investigate the role of miRNA in human mesangial cells (HMCs) stimulated with anti-dsDNA IgG antibodies.

Methods The HMCs were treated with anti-dsDNA IgG antibodies purified from active systemic lupus erythematosus patients or IgG controls in the presence of normal serum for 3 hours. The small RNA expression profile was screened using high throughput sequencing.

Results The results showed that anti-dsDNA IgG up-regulated 103 miRNAs and down-regulated 20 miRNAs which regulate cell cycle, catabolic process, regulation of transcription and apoptosis pathways. Interestingly, miR-10a in HMCs could be validated as specifically down-regulated in HMCs by antidsDNA IgG stimulation. The miR-10a was downregulated in kidney biopsies from lupus nephritis patients and correlated with proteinuria Transiently miR-10a knockdown HMCs increased cells proliferation and up-regulated IL-8 expression. The luciferase assay confirmed that miR-10a down-regulated IL-8 expression by complementary binding to 3'UTR in IL-8.

Conclusions In conclusion, anti-dsDNA IgG Ab down-regulated miR-10a expression in HMC resulting in the induction of various target genes involved in HMCs proliferation as well as inflammation. Manipulation of miR-10a might be a new option for targeted therapy for lupus nephritis.

\section{COMPARISON OF CIRCULATING IMMUNE COMPLEX AND INTERLEUKIN-6 WITH STANDARD BIOMARKERS TO DETERMINE SLE DISEASE ACTIVITY}

${ }^{1} \mathrm{C}$ Thanadetsuntorn*, ${ }^{1} \mathrm{P}$ Ngamjanyaporn, ${ }^{2} \mathrm{C}$ Setthaudom, ${ }^{1} \mathrm{P}$ Pisitkun. ${ }^{1}$ Faculty of Medicine Ramathibodi Hospital, Internal medicine department, Bangkok, Thailand; ' $F a c u l t y$ of Medicine Ramathibodi Hospital, Pathology department, Bangkok, Thailand

\subsection{6/lupus-2017-000215.323}

Background and aims Systemic Lupus Erythematosus (SLE) is an autoimmune disease involving in autoantibody production, immune complex deposition and complement activation. When the disease is active, the sequel can be devastated if inappropriately treated.The outcome of SLE patients can improve if sensitive biomarkers can identify the recent flare and lead the patients to receive the correct treatment in time. This study aimed to investigate whether serum levels of IL-6 and circulating immune complex (CIC) correlated with SLE disease activity and compared with anti-dsDNA and complement.

Methods Ninety SLE patients followed up at Ramathibodi Hospital in 2015 were enrolled. The evaluation of disease activity achieved by Systemic Lupus Erythematosus Disease Activity Index (SLEDAI). The active disease defined if the scores were more than one. Serum IL-6 and CIC tested by ELISA.

Results The level of serum IL-6 and CIC in SLE patients with active disease activity was significantly higher than the inactive

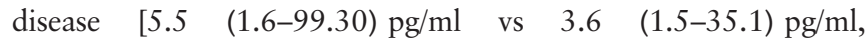
$\mathrm{p}=0.011$ and10.12 (2-131.22) RU/ml vs 2.1 (2.0-101.37) RU/ $\mathrm{ml}, \mathrm{p}=0.011$, respectively]. The correlation analysis between serum biomarkers and clinical SLEDAI demonstrated that biomarkers significantly correlated with SLE activity are CIC $(\mathrm{R}=0.331, \mathrm{p}=0.001)$ and IL-6 $(\mathrm{R}=0.313, \mathrm{p}=0.011)$. CIC had the most area under the curve in discriminating active SLE than IL-6, anti-dsDNA, C4 and C3 (AUC=0.698, 0.677, $0.634,0.410$ and 0.393 respectively) 
Conclusions Serum CIC and IL-6 significantly correlated with clinical SLEDAI, which is higher degree of correlation than anti-dsDNA, C4 and C3 levels. Our study suggested that CIC and IL-6 can be used as alternative biomarkers to determine SLE activity.

\section{IMMUNE COMPLEX GLOMERULONEPHRITIS ASSOCIATED WITH LYSINURIC PROTEIN INTOLERANCE: A CASE REPORT AND REVIEW OF THE LITERATURE}

${ }^{1} \mathrm{MH}$ Tseng*, ${ }^{2} \mathrm{~L}$ Shih-Hua, ${ }^{1} \mathrm{H}$ Jing-Long. 'Chang Gung Memorial Hospital, Paediatrics, TAOYUAN, Taiwan R.O.C; ${ }^{2}$ Tri-Service General Hospital, Medicine, Taipei, Taiwan R.O.C

\subsection{6/lupus-2017-000215.324}

Background and aims Patients with lysinuric protein intolerance (LPI) due to inherited defect of cationic amino acid transport in intestine and renal tubules may have aberrant immune responses leading to multiple organ involvement. The renal involvement with immune complex glomerulonephritis in LPI is albeit rare and has not been well established.

Methods We report a 4-year-old boy manifested nephrotic syndrome with renal histological findings showing immune complex glomerulonephritis highly suggested of lupus nephritis, but the initial serology survey excluded the diagnosis of SLE initially. The diagnosis of lysinuric protein intolerance was established and SLE developed 1 year later. Renal manifestations in patients with LPI and the coexistence of LPI with SLE are reviewed.

Results The initial renal involvement in LPI included renal tubular dysfunction, nephritic and nephrotic syndrome. During follow-up, some patients developed renal function impairment and may progress to end stage renal disease. Glomerulus was the major involved lesion with the most common histological finding was immune complex glomerulonephritis. Five patients, including our patient, with LPI coexisted with SLE have been reported during follow-up. These patients characterised female predominant, young onset age, predominant renal involvement, and poor prognosis. Our patient supported the suggested mechanism of macrophage activation. Treatment with steroid and cyclosporine accordingly led to remission of nephritis.

Conclusions LPI was not only a disorder of amino acid wasting but also a complex multisystemic disease with aberrant immune responses. LPI-associated glomerulonephritis shares similar characteristics on renal histology with lupus nephritis. Both macrophage activation and excess arginine accumulation might play roles on the pathogenesis.

\section{IS LUPUS AN INFLAMMATION RESOLUTION DEFICIENCY DISORDER?}

${ }^{1} D$ Undurti*. ${ }^{1}$ UND Life Sciences LLC, R \& D, Federal way, USA

10.1136/lupus-2017-000215.325

Background and aims Lupus and lupus nephritis progression and flares are difficult to predict. Recently, osteoprotegerin, endothelin-1, $\mathrm{CXCR}^{+}{ }^{+} \mathrm{CD}^{+}{ }^{+} \mathrm{T}$ cells and MCP-1 mRNA expression in urine sediments have been described as possible biomarkers of lupus and lupus nephritis. But their relationship with histological activity has not been sufficiently explored. It is desired that biomarkers of a disease should more rapidly reflect disease progression which would allow shorter clinical proof of concept trials and should be able to predict flares, measure current disease activity and severity, predict progression of disease and prognosis.

Methods It is likely that continued inflammatory events seen in lupus could be due to failure of the resolution of inflammation. Thus, the balance between inflammation and resolution is tilted more in favour of pro-inflammatory events and/ or failure of production of pro-resolution molecules at the most appropriate time leading to non-resolution of inflammation. One such endogenous pro-resolution and anti-inflammatory molecule is lipoxin $\mathrm{A}_{4}$, whose deficiency could lead to continuation of inflammation in lupus and lupus nephritis.

Results It was noted that low plasma and urinary lipoxin $\mathrm{A}_{4}$ indicated disease activity and progression of disease, while a fall in its levels were noted prior to impending flares and increase in disease activity; and an increase in the levels of lipoxin $\mathrm{A}_{4}$ suggested resolution of inflammation and amelioration of disease process.

Conclusions It is suggested that measurement of plasma and urinary lipoxin $\mathrm{A}_{4}$ will be a good biomarker to predict flares, measure current disease activity and severity, predict progression of disease and prognosis.

\section{ROLE OF BIOACTIVE LIPIDS IN AUTOIMMUNE DISEASES INCLUDING LUPUS}

${ }^{1}$ D Undurti*. ${ }^{1}$ UND Life Sciences, $R \& D$, Federal Way, USA

10.1136/lupus-2017-000215.326

Background and aims To evaluate whether a regimen of corticosteroids and cyclophosphamide, and methods designed to enhance endothelial NO synthesis and augment antioxidant defenses can lead to long-lasting remission of lupus and other autoimmune diseases.

Methods Patients with established lupus, dermatomyositis and rheumatoid arthritis are seelcted for the study. Their plasma phospholipid content of unsaturated fatty acids, nitric oxide, anti-oxidants and cytokines are measured before and after treatment.

Results Plasma phospholipid content of arachidonic, eicosapentaenoic and docosahexaenoic acids (AA, EPA and DHA respectively) were found to be low and so also plasma nitric oxide levels and anti-oxidants with a concomitant increase in plasma IL-6 and TNF concentrations. These patients were given pulses of methylprednisolone and cyclophosphamide based on their disease status and response to therapy followed by oral supplementation of GLA/EPA/DHA daily.

Conclusions All patients who entered the study went in to full remission that was found to be associated with restoration of plasma phospholipid content of AA/EPA/DHA, antioxidants and nitric oxide and cytokines to normal. All these patients were in full remission even after stopping immunosuppressive drugs but are continuing GLA/EPA/DHA orally. 